\title{
Pneumothoraces Post Mechanical Cardiopulmonary Resuscitation: A Case Series
}

\author{
Sean Dooley MD and Mohamed Mohsen Mansour MD
}

\begin{abstract}
The incidence of pneumothoraces with automated cardiopulmonary resuscitation (CPR) is unknown. Herein, we present 4 cases of pneumothoraces occurring in the setting of automated mechanical CPR (AM-CPR) in a 2-month period since incorporating mechanical devices into our resuscitation program. Two of the cases were in-hospital cardiac arrests, whereas the other 2 were out-of-hospital cardiac arrests. The Life-Stat 1008 device was utilized for AM-CPR in all cases. All cases demonstrated confirmed pneumothoraces on post-resuscitation imaging. Several factors may have contributed to the observed pneumothoraces. Two of the cases presented with obstructive lung disease, whereas the other 2 had underlying malignancy. Suboptimal positioning and failure to secure the included shoulder straps could have led to migration of the piston over the ribs. Further study is needed to determine the incidence of complications for all FDA-approved AM-CPR devices compared with manual chest compressions. Key words: resuscitation; pneumothorax; CPR; automated mechanical CPR; Life-Stat 1008; cardiac arrest. [Respir Care 2015;60(7):e118-e121. (C) 2015 Daedalus Enterprises]
\end{abstract}

\section{Introduction}

With the revision of the American Heart Association cardiopulmonary resuscitation (CPR) guidelines in 2010 and the emphasis on adequate chest compressions, ${ }^{1}$ many have turned to automated mechanical CPR (AM-CPR) devices as a way to standardize compressions. Although AMCPR devices were initially introduced in 1908, they were not widely utilized until the 1960s. ${ }^{2}$ In the United States, there are 3 different AM-CPR devices cleared by the FDA. The Life-Stat 1008 (Michigan Instruments, Grand Rapids, Michigan) employs a piston-like system to depress the sternum, whereas the AutoPulse 100 (Zoll Medical, Chelmsford, Massachusetts) utilizes a load-distributing

Dr Dooley is affiliated with the Division of Internal Medicine, and Dr Mansour is affiliated with the Division of Pulmonary and Critical Care, Department of Medicine, Stony Brook University Hospital, Stony Brook, New York.

The authors have disclosed no conflicts of interest.

Correspondence: Mohamed Mohsen Mansour MD, Stony Brook University Hospital, HSC T17-040, Stony Brook, NY 11794-8172. E-mail: mohamed.mansour@stonybrookmedicine.edu.

DOI: $10.4187 /$ respcare. 03334 band system (LifeBand) to distribute compressive force across the chest wall (Fig. 1). In contrast, the Lucas 2 (Physio-Control/Jolife, Lund, Sweden) utilizes a suctioncup system to actively compress and decompress the chest. AM-CPR devices may be advantageous in reducing variation in chest compressions, eliminating rescuer fatigue, and minimizing interruptions during CPR, permitting ongoing rhythm evaluations and defibrillation. ${ }^{2,3}$ They also allow for prolonged resuscitation, with documented success after $80 \mathrm{~min}$ in one case report. ${ }^{4}$ AM-CPR devices have been found to improve coronary and cerebral perfusion pressures ${ }^{5-7}$ and return of spontaneous circulation in out-of-hospital cardiac arrests, ${ }^{8}$ although this has not withstood systematic review. ${ }^{9-11}$

Complications associated with mechanical CPR devices are often assumed to be similar to those associated with manual CPR, and studies comparing head-to-head complication rates are sparse. Smekal et $\mathrm{al}^{12}$ found no difference in autopsy-detected injuries in 85 cardiac arrest subjects treated with manual compressions compared with mechanical compressions with the Lucas 2 . However, Pinto et al ${ }^{13}$ found that manual compressions were associated with anterior rib and sternal fractures in contrast to a higher frequency of posterior rib and vertebral fractures with the AutoPulse 100 along with a few reported cases of liver and splenic lacerations causing hemoperitoneum. Studies re- 


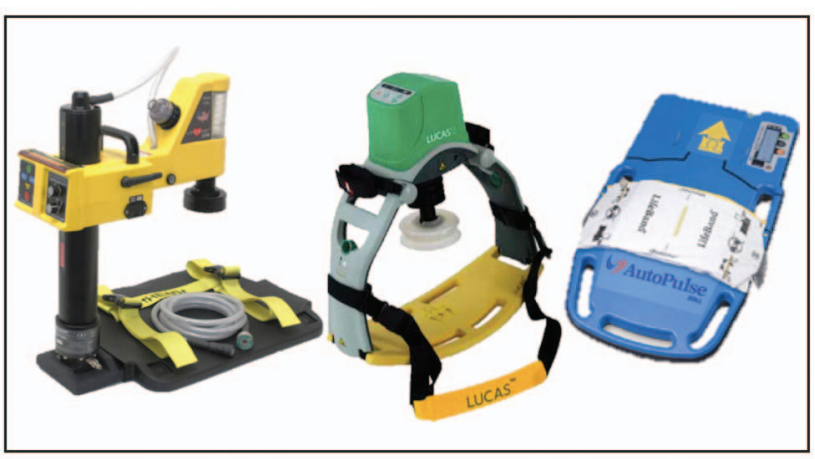

Fig. 1. Automated mechanical cardiopulmonary resuscitation (AMCPR) devices. From left to right: Life Stat 1008 (Michigan Instruments), AutoPulse 100 (Zoll Medical), and Lucas 2 (Physio Control/Jolife).

ported increased thoracic injury, including rupture of all 4 heart chambers and aorta, attributable to AM-CPR devices. ${ }^{14-16}$ Although rib and sternal fractures are considered common complications of manual CPR, ranging from 13 to $97 \%$ and from 1 to $43 \%$, respectively, pneumothoraces are infrequently described and are cited at an incidence of $\sim 1-3 \% .{ }^{17}$ Data for AM-CPR-associated pneumothoraces are lacking. We could find only one case report available in the literature of a tension pneumothorax after resuscitation with a AM-CPR device. ${ }^{18}$ We present here 4 cases of pneumothoraces that occurred after resuscitation with an AM-CPR device at our institution over a 2-month period.

\section{Case Reports}

\section{Case 1}

An 80-y-old female presented to the emergency department with complaints of abdominal pain and a fever of $38.9^{\circ} \mathrm{C}$. She was found to be hypotensive with a positive urinalysis. Computed tomography of the abdomen and pelvis performed in the emergency department demonstrated an incidental $3-\mathrm{cm}$ mass in the right paracolic gutter with metastases to liver, brain, and bone, including an anterior mediastinal mass eroding into the left posterior sternum. An echocardiogram was ordered because of positive troponins in the setting of a normal electrocardiogram and demonstrated severe right ventricular enlargement with hypokinesis, suggesting pulmonary embolism. During the workup, the patient developed sudden progressive bradycardia and suffered a pulseless electrical activity arrest. The Life-Stat 1008 was utilized for resuscitation as per institutional protocol following the immediate but brief commencement of manual compressions while the device was being set up. The patient was intubated and resuscitated for $\sim 1 \mathrm{~h}$ with eventual return of spontaneous circu-

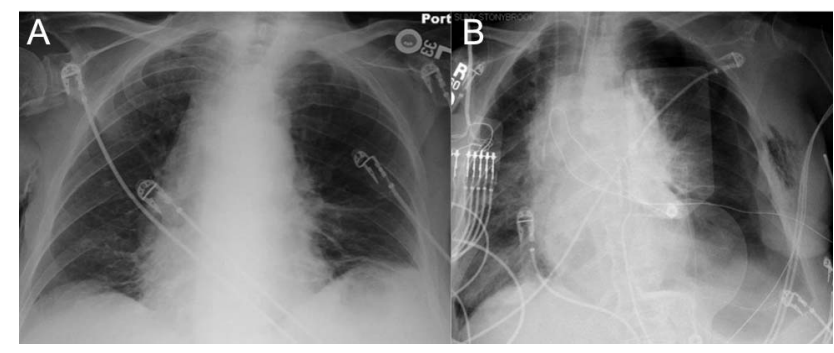

Fig. 2. Case 1 portable supine chest radiograph pre-resuscitation (A) and post-resuscitation (B).

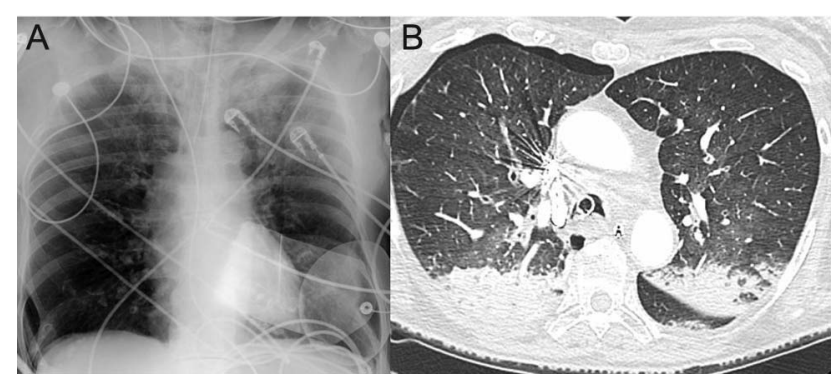

Fig. 3. Case 2 portable supine chest radiograph and computed tomography imaging post-resuscitation demonstrating a large, right-sided anterior pneumothorax and numerous bilateral rib fractures (A: 2nd-7th ribs; B: 1st-9th ribs).

lation. A post-resuscitation chest radiograph to check the placement of a right-sided internal jugular central venous catheter revealed a new, moderate, left-sided pneumothorax with evidence of shift, suggesting a tension pneumothorax (Fig. 2). A left-sided internal jugular or subclavian line was never attempted, and prior imaging had revealed no pneumothorax.

\section{Case 2}

An 86-y-old female with poorly controlled asthma collapsed after she awoke with worsening shortness of breath. Her son called emergency medical services, who arrived and found the patient in pulseless electrical activity arrest. An AutoPulse 100 device was utilized in the field for resuscitation. The patient was brought to the emergency department and transitioned to a Life-Stat 1008. Sustained return of spontaneous circulation was eventually achieved after a total resuscitation time of $\sim 40 \mathrm{~min}$. A chest radiograph and chest computed tomography obtained postresuscitation demonstrated a new, large, right-sided anterior pneumothorax without tension, as well as numerous bilateral rib fractures involving the 1st-9th ribs on the right and the 2nd-7th seventh ribs on the left (Fig. 3).

\section{Case 3}

A 73-y-old female with a history of severe COPD and atrial fibrillation was brought to the emergency depart- 


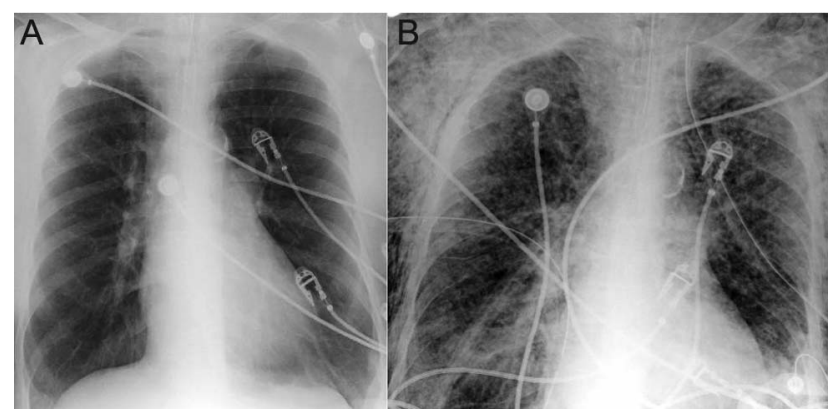

Fig. 4. Case 3 portable supine chest radiograph pre-resuscitation (A) and post-resuscitation (B) demonstrating extensive subcutaneous emphysema, bilateral chest tubes, and a small, right-sided apical pneumothorax.

ment by her son after 2 days of worsening dyspnea and altered mental status. The patient was found to be in hypoxemic and hypercapnic respiratory failure and was subsequently intubated. A post-intubation chest radiograph was normal (Fig. 4). Shortly after being sedated, the patient developed progressive bradycardia and went into pulseless electrical activity arrest. CPR was begun with a Life-Stat 1008 device, and return of spontaneous circulation was initially achieved after $\sim 10 \mathrm{~min}$. This was followed by a subsequent arrest, during which marked subcutaneous emphysema was noted throughout the anterior chest wall bilaterally associated with high peak pressures on the ventilator. Immediate needle decompression was performed, and chest tubes were placed bilaterally. Peak pressures improved, and sustained return of spontaneous circulation was achieved after $9 \mathrm{~min}$. A post-resuscitation chest radiograph demonstrated extensive bilateral subcutaneous emphysema with a residual right-sided pneumothorax (Fig. 4).

\section{Case 4}

A 42-y-old female with a history of multiple myeloma, systemic lupus erythematosus, and chronic kidney disease was admitted with headaches and photophobia. During her hospital stay, a rapid response was called for unresponsiveness requiring emergent intubation for airway protection. A post-intubation portable chest radiograph demonstrated that the endotracheal tube and left-sided internal jugular line were in proper position (Fig. 5). There was no pneumothorax after placement of the internal jugular line, and no subclavian line was attempted. The patient continued to rapidly deteriorate, suffered a pulseless electrical activity arrest, and was resuscitated with a Life-Stat 1008. Return of spontaneous circulation was achieved after $\sim 9$ min. A post-resuscitation portable chest radiograph demonstrated a large, right-sided tension pneumothorax (Fig. 5).

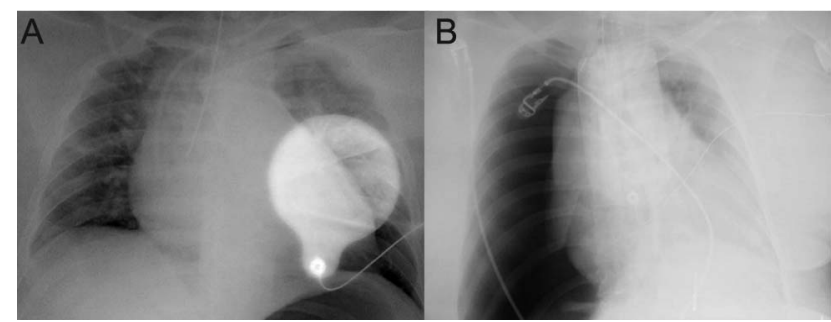

Fig. 5. Case 4 portable supine chest radiograph pre-resuscitation (A) and post-resuscitation (B) demonstrating a large, right-sided tension pneumothorax.

\section{Discussion}

Over the past year, the Life-Stat 1008 has been adopted as the resuscitation method of choice at our institution owing to the hemodynamic improvements in cerebral and coronary perfusion pressures and the ability to eliminate variability in compressions and rescuer fatigue. The discovery of these 4 post AM-CPR pneumothoraces raised the question of comparative complication rates between automated and manual CPR. Pneumothoraces can be an important cause of post-resuscitation morbidity and mortality if not suspected by the clinician. Although deemed an infrequent complication of manual CPR, ${ }^{17}$ the incidence with AM-CPR is not known. In general, the complication rates of automated CPR devices compared with manual CPR devices are not well described.

Further complicating the matter is that generalizations across all 3 device types may be inaccurate, as they vary mechanistically. The 2 autopsy-based retrospective studies listed above demonstrated different injury patterns for different devices. This may represent the difference between how force is applied to the sternum and chest wall (ie, over a focal area, as in the piston-driven system, or across the entirety of the chest wall, as in the banded system). The AutoPulse 100 and Thumper 1004 (Michigan Instruments) were compared in a porcine experimental model: more rib fractures were found with the Thumper. ${ }^{19}$ This, as well as other device-specific factors such as noncompliance with application of accompanying shoulder straps, improper positioning on the small proprietary backboard, and device migration, may in fact increase the risk of a post AM-CPR pneumothorax compared with manual CPR.

Inability to position patients properly can cause suboptimal alignment of the piston over the sternum and lead to migration of the device off the sternum. Piston migration across the chest during resuscitation may cause pressure to be applied directly over the ribs instead of the sternum and lead to higher rates of rib fractures and pneumothoraces. The various AM-CPR devices may not migrate to the same degree and may distribute forces differently across the chest. Application of shoulder straps can be time-consum- 


\section{Pneumothoraces Post Mechanical CPR}

ing, can delay chest compressions, and may not be possible due to patient body habitus.

A patient's comorbid conditions may increase the risk of developing pneumothoraces as well. In our first case, the patient's sternal integrity was compromised given the erosive metastatic lesion. Two other cases involved patients with obstructive lung disease presenting with exacerbations. Hyperinflation can theoretically increase the risk of developing a pneumothorax, especially with a migrating device depressing fractured ribs into the lungs.

This case series has some limitations. First, 3 of the 4 cases had a pre-resuscitation portable supine chest radiograph. Small pneumothoraces may not be visualized well in the supine position, although larger ones will be indicated by the deep sulcus sign. The second case report was a pre-hospital arrest, and imaging was not performed before chest compressions. Finally, in some cases before the AM-CPR device is used, there is a variable period of manual chest compressions before placement of the device.

AM-CPR devices show promise with regard to hemodynamic parameters and outcomes in resuscitation. Further study is needed to determine whether the incidence of pneumothoraces and other complications is increased with use of mechanical devices compared with manual CPR and to compare the incidence among the 3 different devices. Whether certain presenting conditions may be relative contraindications to the use of automated compression devices also still needs to be determined. Increased vigilance for pneumothorax may be needed post-resuscitation with AM-CPR devices.

\section{REFERENCES}

1. Neumar RW, Otto CW, Link MS, Kronick SL, Shuster M, Callaway CW, et al. Part 8: adult advanced cardiovascular life support: 2010 American Heart Association Guidelines for Cardiopulmonary Resuscitation and Emergency Cardiovascular Care. Circulation 2010; 122(18 Suppl 3):S729-S767.

2. Wigginton JG, Miller AH, Benitez FL, Pepe PE. Mechanical devices for cardiopulmonary resuscitation. Curr Opin Crit Care 2005;11(3): 219-223.

3. ZOLL AutoPulse resuscitation system model 100 user guide. http:// www.zoll.com/WorkArea/DownloadAsset.aspx $? \mathrm{id}=21383$. Accessed December 26, 2014.

4. Piacentini A, Volonte M, Rigamonti M, Guastella E, Landriscina M. Successful prolonged mechanical CPR in a severely poisoned hypo- thermic patient: a case report. Case Rep Emerg Med 2012;2012: 381798.

5. Ikeno F, Kaneda H, Hongo Y, Sakanoue Y, Nolasco C, Emami S, et al. Augmentation of tissue perfusion by a novel compression device increases neurologically intact survival in a porcine model of prolonged cardiac arrest. Resuscitation 2006;68(1):109-118.

6. Timerman S, Cardoso LF, Ramires JA, Halperin H. Improved hemodynamic performance with a novel chest compression device during treatment of in-hospital cardiac arrest. Resuscitation 2004;61(3): 273-280.

7. Rubertsson S, Karlsten R. Increased cortical cerebral blood flow with LUCAS; a new device for mechanical chest compressions compared to standard external compressions during experimental cardiopulmonary resuscitation. Resuscitation 2005;65(3):357-363.

8. Westfall M, Krantz S, Mullin C, Kaufman C. Mechanical versus manual chest compressions in out-of-hospital cardiac arrest: a metaanalysis. Crit Care Med 2013;41(7):1782-1789.

9. Jennings PA, Harriss L, Bernard S, Bray J, Walker T, Spelman T, et al. An automated CPR device compared with standard chest compressions for out-of-hospital resuscitation. BMC Emerg Med 2012; 12:8.

10. Ong ME, Mackey KE, Zhang ZC, Tanaka H, Ma MH, Swor R, Shin SD. Mechanical CPR devices compared to manual CPR during outof-hospital cardiac arrest and ambulance transport: a systematic review. Scand J Trauma Resusc Emerg Med 2012;20:39.

11. Brooks SC, Bigham BL, Morrison LJ. Mechanical versus manual chest compressions for cardiac arrest. Cochrane Database Syst Rev 2011(1):CD007260.

12. Smekal D, Johansson J, Huzevka T, Rubertsson S. No difference in autopsy detected injuries in cardiac arrest patients treated with manual chest compressions compared with mechanical compressions with the LUCAS device-a pilot study. Resuscitation 2009;80(10):11041107.

13. Pinto DC, Haden-Pinneri K, Love JC. Manual and automated cardiopulmonary resuscitation (CPR): a comparison of associated injury patterns. J Forensic Sci 2013;58(4):904-909.

14. Nelson BK. Tension pneumothorax following CPR or mechanical ventilation. Ann Emerg Med 1985;14(6):615.

15. Klintschar M, Darok M, Radner H. Massive injury to the heart after attempted active compression-decompression cardiopulmonary resuscitation. Int J Legal Med 1998;111(2):93-96.

16. Baubin M, Sumann G, Rabl W, Eibl G, Wenzel V, Mair P. Increased frequency of thorax injuries with ACD-CPR. Resuscitation 1999; 41(1):33-38.

17. Hoke RS, Chamberlain D. Skeletal chest injuries secondary to cardiopulmonary resuscitation. Resuscitation 2004;63(3):327-338.

18. Hutchings AC, Darcy KJ, Cumberbatch GL. Tension pneumothorax secondary to automatic mechanical compression decompression device. Emerg Med J 2009;26(2):145-146.

19. Chen W, Weng Y, Wu X, Sun S, Bisera J, Weil MH, Tang W. The effects of a newly developed miniaturized mechanical chest compressor on outcomes of cardiopulmonary resuscitation in a porcine model. Crit Care Med 2012;40(11):3007-3012. 